\title{
Special issue on the intelligent sensing on mobile computing and wireless communications
}

\author{
Zheng $X u^{1} \cdot$ Yunhuai Liu ${ }^{2} \cdot$ Neil Y. Yen ${ }^{3}$
}

Published online: 15 March 2018

c) Springer-Verlag GmbH Germany, part of Springer Nature 2018

Nowadays, it has become convenient to capture images and videos on the mobile end and associate them with social and contextual metadata such as comments and GPS tags (Xu et al. 2018). Such a hybrid data structure can benefit a wide variety of potential applications on the mobile end, such as location recognition, landmark search, augmented reality, and commercial recommendations (Xu et al. 2017). Current research in Intelligent Sensing addresses the following issues: Intelligent Sensing as a novel methodology for user-centered research; development of new services and applications based on human sensing, computation, and problem solving; engineering of improved Intelligent Sensing platforms including quality control mechanisms; incentive design of work; usage of Participatory Sensing for professional business; and theoretical frameworks for evaluation (Xu et al. 2017a, b, c, d). This is opening a vast space of opportunities to extend the current networks, communications, and computer applications to more pervasive and mobile applications.

The submitted manuscripts were reviewed by experts from both academia and industry. After two rounds of reviewing, the highest quality manuscripts were accepted for this special issue. Totally, we have received 22 manuscripts and 14 papers are accepted. This special issue will publish by Journal of Ambient Intelligence and Humanized Computing as special issues. We also balance the region of accepted papers. Authors are come from China, USA, Sweden, and Tanzania.

Wang et al. (2017) propose a low-complexity, partial priori information-sparsity adaptive matching pursuit (PPI-SAMP) algorithm. Unlike the conventional SAMP

Zheng Xu

juven_xz@163.com

1 The third research institute of the ministry of public security, Shanghai 201142, China

2 Peking University, Beijing, China

3 University of Aizu, Aizuwakamatsu, Japan algorithm, the PPI-SAMP algorithm improves performance over fast-fading channels by adequately exploiting the sparse characteristics and temporal correlation of wireless channels. Focusing on weighted throughput fairness that nodes get throughput proportions according to priority levels in IEEE 802.11e WLANs, Shi et al. (2017) propose an adaptive and full-distributed access mechanism with delta estimation algorithm of traffic loads. Zhang et al. (Yong et al. 2017) proposed a gas leakage source localization algorithm using distributed maximum likelihood estimation method for mobile sensor network to improve the lower performance with static sensor network. In order to achieve higher gains and lower side lobe in antenna design, array antenna is conventionally adopted by $\mathrm{Li}$ et al. (Wei et al. 2017). Zheng et al. (2018) present a Parallel Gated Poll (PGP) access mechanism that exploits the PCF defined in the IEEE 802.11. To improve the transmission efficiency of content dissemination, Zhang et al. (2017) propose an efficient caching scheme for ICN, which is named as NCPP-based caching scheme. Chen et al. (2017) regard the signed network as an identical-discrepancy-contrary system employing the set pair theory, and propose a new link prediction measure SNCD which integrates both the certain and uncertain relations, local and global information at the same time. Based on the DSR, the stability of the link is estimated by Yang et al. (2017) using Continuous Hopfield Neural Network to find the route with the highest stability from the source node to the destination node to improve the performance of the DSR and improve the performance of the MANET. Combined with angular domain channel sparsity, compressive sensing (CS) technique is introduced by Wang et al. (2017a, b) to estimate downlink CSI because it can help the frequency division duplex massive MIMO system to overcome the restriction of the limited pilot overhead. Huang et al. (2017) propose novel sub-band spectral centroid weighted wavelet packet Cepstral coefficients (W-WPCC) for robust speech emotion recognition. With the method of alternating-direction-implicit finite-difference time-domain (ADI-FDTD), 
Wu et al. (2018) discuss the logging response of electromagnetic wave LWD in deviated well. Li and Sugumaran (Li et al. 2018) propose a cognitive-based AES (CAES) model to deal with these issues. It is composed of three parts: sensory acquisition, score analyzer and background knowledge constructor. Zhang et al. (2018a, b) present a new trapdoor function based on "ring learning with errors" problem (Ring-LWE) on lattice, and simultaneously the corresponding efficient inverse algorithm is given which involves two sub-algorithms: the trapdoor inverse algorithm and the iterative inverse algorithm. Zhang et al. (2018) studied the mainstream node load balance technologies and proposed an evaluation model which identifies and measures the level of load.

Acknowledgements The guest editors would like to thank Prof. Vincenzo Loia who is the editor in chief of Journal of Ambient Intelligence and Humanized Computing. His help and trust is the most important thing for the success of this SI. The guest editors would like to thank the reviewers for their high quality reviews, which provided insightful and constructive feedback to the authors of the papers.

\section{References}

Chen X, Guo JF, Pan X et al (2017) Link prediction in signed networks based on connection degree. J Ambient Intell Human Comput. https://doi.org/10.1007/s12652-017-0613-2

Huang Y, Tian K, Wu A et al (2017) Feature fusion methods research based on deep belief networks for speech emotion recognition under noise condition. J Ambient Intell Human Comput. https:// doi.org/10.1007/s12652-017-0644-8

Li L, Sugumaran VA, cognitive-based (2018) AES model towards learning written English. J Ambient Intell Human Comput. https ://doi.org/10.1007/s12652-018-0743-1

Shi C, He SQ, Deng ZJ et al (2017) Adaptive access mechanism with delta estimation algorithm of traffic loads for supporting weighted priority in IEEE 802.11e WLANs. J Ambient Intell Human Comput. https://doi.org/10.1007/s12652-017-0588-Z

Wang W, Xiu Y, Zhang Z (2017a) FDD massive MIMO downlink channel estimation with complex hybrid generalized approximate message passing algorithm. J Ambient Intell Human Comput. https://doi.org/10.1007/s12652-017-0634-x

Wang R, Cai J, Yu X et al (2017b) Temporal-correlation-based compressive channel estimation for universal filtered multicarrier system over fast-fading channels. J Ambient Intell Human Comput. https://doi.org/10.1007/s12652-017-0593-2

Wei L, Changwu X, Yue H et al (2017) Actual deviation correction based on weight improvement for 10-unit Dolph-Chebyshev array antennas. J Ambient Intell Human Comput. https://doi. org/10.1007/s12652-017-0589-y

Wu A, Fu Q, Mwachaka SM et al (2018) Numerical modeling of electromagnetic wave logging while drilling in deviated well. J Ambient Intell Human Comput. https://doi.org/10.1007/s1265 2-018-0700-Z

Xu Z, Yen NY, Zhang H, Wei X, Lv Z, Choo KR, Mei L X. Luo (2017a) Social Sensors Based Online Attention Computing of Public Safety Events. IEEE Trans Emerging Topics Comput 5(3):403-411

Xu Z, Zhang H, Hu C, Liu Y, Xuan J, Mei L (2017b) Crowdsourcingbased timeline description of urban emergency events using social media. IJAHUC 25(1/2):41-51

Xu Z, Liu Y, Zhang H, Luo X, Mei L C. Hu. (2017c) Building the multi-modal storytelling of urban emergency events based on crowdsensing of social media analytics. MONET 22(2):218-227

Xu Z, Liu Y, Xuan J, Chen H, Mei L (2017d) Crowdsourcing based social media data analysis of urban emergency events. Multimedia Tools Appl 76(9):11567-11584

Xu Z, Liu Y, Mei L, Luo X, Hu C, Zhang H J. Yu (2017e) The mobile media based emergency management of web events influence in cyber-physical space. Wireless Pers Commun 95(3):1877-1890

Xu Z, Mei L, Choo KR, Lv Z, Hu C, Luo X, Liu Y (2018) Mobile crowd sensing of human-like intelligence using social sensors: a survey. Neurocomputing 279:3-10

Yang H, Li Z, Liu Z (2017) A method of routing optimization using CHNN in MANET. J Ambient Intell Human Comput. https://doi. org/10.1007/s12652-017-0614-1

Yong Z, Liyi Z, Jianfeng H et al (2017) An indoor gas leakage source localization algorithm using distributed maximum likelihood estimation in sensor networks. J Ambient Intell Human Comput. https ://doi.org/10.1007/s12652-017-0624-Z

Zhang M, Xie P, Zhu J et al (2017) NCPP-based caching and NURbased resource allocation for information-centric networking. J Ambient Intell Human Comput. https://doi.org/10.1007/s1265 2-017-0590-5

Zhang C, Ma W, Zhao F (2018a) Trapdoor function based on the Ring-LWE and applications in communications. J Ambient Intell Human Comput. https://doi.org/10.1007/s12652-018-0718-2

Zhang W, He ZY, Ma LM et al (2018b) AP load balance strategy in face of high user density. J Ambient Intell Human Comput. https ://doi.org/10.1007/s12652-018-0690-x

Zheng G, Zhi-Jun Y, Min H et al (2018) Energy-efficient analysis of an IEEE 802.11 PCF MAC protocol based on WLAN. J Ambient Intell Human Comput. https://doi.org/10.1007/s12652-018-0684-8 